\title{
Rectal Juvenile Polyp
}

National Cancer Institute

\section{Source}

National Cancer Institute. Rectal Juvenile Polyp. NCI Thesaurus. Code C5618.

A non-neoplastic hamartomatous polyp that arises from the rectum. It is characterized by the presence of tortuous and cystically dilated glands, edematous changes, and inflammation. 